\title{
Diabetes and the Heart - a Never-ending Story
}

\author{
Rolf Dörr', Dirk Müller-Wieland², Diethelm Tschoepe ${ }^{3}$
}

\author{
${ }^{1}$ Practice Clinic Heart \\ and Vessels, Dresden, \\ Germany \\ ${ }^{2}$ Asklepios Hospital \\ St. Georg, Hamburg, \\ Germany \\ ${ }^{3}$ Ruhr University, \\ Bochum, Germany
}

It is now, after 2004 and 2008, the third issue of the renowned journal Herz dedicated to the topic of "Diabetes and the Heart", justified by the emerging epidemiologic catastrophe with exploding numbers of patients affected by both sides of metabolic and vascular medicine. After the landmark publication of a joint guideline between two European scientific societies highlighting the scientific knowledge and the care status of patients with diabetes and cardiac manifestations, we ended with the conclusion that despite advances in the cardiovascular field new forms of heart disease such as heart failure or atrial fibrillation have come into focus. Moreover, the interplay between cardiovascular disease, diabetes and associated comorbidities such as obesity, depression or obstructive sleep apnea has been addressed, suggesting that adequate diagnosis and related therapeutic interventions will significantly improve quality of life and the prognosis of affected patients. Moreover, the frame and the estimates of the prognosis-limiting factors have changed such as the question of morbid obesity. The issue of benefit versus risk has been raised both in the long-term outpatient treatment for normoglycemia as well as for perioperative inpatient glucose control or Dronedarone for rhythm control, allowing a more proper adjustment between risk and benefit in individual patients. So, this issue of the journal tries to update the clinical data base around patients with diabetes and heart problems. Pathophysiological

considerations around metabolic contributions both to diabetic cardiomyopathy as well as cardiovascular disease are addressed as well as hot clinical questions such as "appropriate glucose control", "surgery or PCI" or "criteria of a specific diabetes-related cardiomyopathy" are discussed. With this agenda the journal Herz furthers a tradition of scientific dispute as a platform for better decision-making of doctors taking care of patients with both diabetes and heart problems.
Herz 2010;35:129

DOI 10.1007/ s00059-010-3343-1

\section{Address for Correspondence}

Dr. Rolf Dörr

Praxisklinik Herz und Gefäße

Forststraße 3

1099 Dresden

Germany

Phone (+49/351) 2693-6505,

Fax -6506

e-mail:doerr@praxisklinik-dresden.de 